\title{
Faunistic study on Lepidoptera from different regions of Anatolia, Turkey
}

\author{
Yusuf Hüseyinoğlu \\ Department of Biology, Faculty of Science and Letters, Mersin University, Mersin, Turkey
}

\section{Email address:}

yusufan2000@yahoo.com (Y. Hüseyinoğlu)

\section{To cite this article:}

Yusuf Hüseyinoğlu. Faunistic Study on Lepidoptera from Different Regions of Anatolia, Turkey, Animal and Veterinary Sciences. Vol. 1, No. 1, 2013, pp. 7-11. doi: 10.11648/j.avs.20130101.12

\begin{abstract}
This study was carried out in Ankara, Antalya, Denizli, Isparta, Istanbul, Kırşehir, Konya, Malatya, Nevşehir and Ordu (Turkey), during the years of 2003-2006 in order to collect data on Papilionoidea and Hesperioidea species, their distribution and current status. Butterflies samples were caught with sweep nets from 18 localities during the field work. 77 species belonging to 6 families and 2 super families were identified. According to identification results the numbers of species (belonging to families) were given as: Papilionidae (3); Pieridae (13); Argynnidae (12); Satyridae (22); Lycaenidae (22) and Hesperiidae (5).
\end{abstract}

Keywords: Lepidoptera, Papilionoidea, Hesperioidea, Fauna, Anatolia, Turkey

\section{Introduction}

Butterflies are the Charismatic Megafauna of the Insect World. The wings of butterflies are colored and patterned in such a way that the majority of species can be identified on sight. They are holometabolous; that is, they have four distinct stages in their life cycle: egg, caterpillar or larva, the chrysalis or pupa, and adult (Schappert, 2005; Cassie, 2007; Canakcioglu, 1995; Canakcioglu and Mol, 1998). According to Tolman and Lewington, (1997), in view of the importance of camouflage as a survival strategy, it is not surprising that the behaviour of a butterfly is often highly correlated with its physical appearance and the character of its habitat (Kaygin et al., 2009).

Although a lot of studies about Lepidoptera fauna in Turkey, mostly regional have been carried out but Turkey's Lepidoptera fauna are not complete yet. There are several regional studies about Lepidoptera fauna in Turkey (Atay and Yolcu, 2012; Ayberk et al., 2010; Can, 2008; Hüseyinoğlu, 2000; Kaygin et al., 2009; Koçak and Seven, 1994, 1995; Koçak and Kemal, 2008, 2009, 2012; Kudrna, 1983; Lederer, 1855; Okyar and Aktaç, 1998). Hesselbarth et al. (1995) have declared that there are 345 species in Turkey.

\section{Main Body}

This study was conducted between years 2003 and 2006 for determination fauna of Lepidoptera in Anatolia. The researches have been carried out as field and laboratoary studies. The field studies carried out in Ankara, Antalya, Denizli, Isparta, Istanbul, Kırşehir, Konya, Malatya, Nevşehir and Ordu during May- August in 18 localities and different habitat types between altitudes from 30 to $1300 \mathrm{~m}$. Specimens were collected using a sweep net. Collected specimens were killed in killing jars with ethyl acetate. During the collection, the collection date, name of the locality and altitude were noted on a piece of envelope for every specimen. Each specimen was then put into this envelope and all materials were brought to the laboratory for preparation and identifications. All the butterfly specimens were pinned with wings spread, dried, and put into collection boxes. For identification of specimens, different studies such as Hesselbarth et al., (1995), Hofmann and Marktanner (1995), Tolman and Lewington (1997) and author's collections of comparative materials were used.

\section{Results}

A total of 77 species belonging to 6 families and 2 super families of the order Lepidoptera were identified. The highest number of species belongs to Satyridae (22, 28.6\%), and Lycaenidae $(22,28.6 \%)$, followed by Pieridae (13, $16.8 \%$ ), Argynnidae (12, 15.6\%), Hesperidae (5, 6.4\%), and Papilionidae $(3,4 \%)$. The number of species in each family and their ratios to the families were given in Table 1. 
Table 1. Number of species and rates of families in Anatolia.

\begin{tabular}{lll}
\hline Family & Number of species & Rate (\%) \\
\hline Papilionidae & 3 & 4 \\
Pieridae & 13 & 16.8 \\
Argynnidae & 12 & 15.6 \\
Satyridae & 22 & 28.6 \\
Lycaenidae & 22 & 28.6 \\
Hesperidae & 5 & 6.4 \\
Total & 77 & 100 \\
\hline
\end{tabular}

The systematic list of species has been formed according to Koçak and Kemal (2009). The capturing dates and the collecting locations about each specimen have been arranged as follows.

\section{Order: LEPIDOPTERA}

Suborder: RHOPALOCERA

Superfamily: PAPILIONOIDEA Latreille, (1802)

Family: PAPILIONIDAE Latreille, (1802)

Iphiclides podalirius (Linnaeus, 1758)

Antalya Thermossus National park 900m. 27.07.2003; Ankara Ayaş Sinanlı 1100m. 01.08.2003; Denizli Maymun Mountains Göz Location 900m. 20.07.2003; Denizli Acıgöl vicinity $850 \mathrm{~m}$. 24.07.2003; Istanbul Şile Bozgoca village 30m. 21.07.2005; Nevşehir Hacıbektaş 1300m. 18.07.2004; Ordu Fatsa Baba village 500m. 28.08.2006.

\section{Papilio machaon Linnaeus, 1758}

Isparta Eğirdir Boyalıköy 900m. 19.05.2004; Ankara Batıkent Yuva village 900m. 02.07.2003; Ankara Ayaş beli 1150m. 06.07.2004; Denizli Acıgöl vicinity $850 \mathrm{~m}$. 24.07.2003; Istanbul Şile Bozgoca village 50m. 25.08.2005. Zerynthia (Allancastria) deyrollei (Oberthür, 1869)

Isparta Eğirdir Boyalıköy 900m. 19.05.2004; Malatya

Akçadağ ikinciler village500m. 25.05.2006.

Family: PIERIDAE Duponchel, (1835)

Aporia crataegi (Linnaeus, 1758)

Isparta Eğirdir Boyalıköy 900m. 19.05.2004; Ankara Ayaş beli 1150m. 06.07.2004; Konya Akşehir sorkun 960m. 22.06.2004; Konya Akşehir sorkun 960m. 02.07.2004.

Colias crocea (Fourcroy, 1785)

Antalya Thermossus National park 900m. 27.07.2003; Isparta Eğirdir Boyalıköy 900m. 19.05.2004; Ankara Batıkent Yuva village 900m. 02.07.2003; Ankara Ayaş Sinanl1 1100m. 01.08.2003; Ankara Ayaş beli 1150m. 06.07.2004; Denizli Acıgöl vicinity 850m. 24.07.2003; Istanbul Şile Bozgoca village 30m. 21.07.2005; Kırşehir Aşıkpaşa Atatürk forest 980m. 20.06.2004; Konya Akşehir sorkun 960m. 22.06.2004; Konya Akşehir sorkun 960m. 02.07.2004; Nevşehir Hacıbektaş 1300m. 18.07.2004; Ordu Fatsa Baba village $500 \mathrm{~m}$. 28.08.2006.

Colias (Neocolias) aurorina Herrich-Schaffer, [1804]

Istanbul Şile Bozgoca village 30m. 21.07.2005.

Colias alfacariensis Ribber, 1905

Isparta Eğirdir Boyalıköy 900m. 19.05.2004; Ankara

Ayaş beli 1150m. 06.07.2004.

Euchloe ausonia (Hübner, [1804])

Konya Akşehir sorkun 960m. 22.06.2004.
Gonepteryx farinosa (Zeller, 1847)

Ordu Fatsa Baba village 500m. 28.08.2006.

Leptidea duponcheli (Staudinger, 1871)

Kırşehir Aşıkpaşa Atatürk forest 980m. 20.06.2004; Kırşehir Aşıkpaşa Atatürk forest 980m. 27.06.2004; Kırşehir Aşıkpaşa Atatürk forest 980m. 04.07.2004.

Leptidea sinapis (Linnaeus, 1758)

Isparta Eğirdir Boyalıköy 900m. 19.05.2004; Ankara Ayaş Sinanlı 1100m. 01.08.2003; Denizli Maymun Mountains Göz Location 900m. 20.07.2003.

Pieris brassicae (Linnaeus,1758)

Antalya Thermossus National park 900m. 27.07.2003; Isparta Eğirdir Boyalıköy 900m. 19.05.2004; Istanbul Şile Bozgoca village 30m. 21.07.2005; Istanbul Şile Bozgoca village $30 \mathrm{~m}$. 08.08.2005; Istanbul Şile Bozgoca village 50m. 25.08.2005; Malatya Akçadağ ikinciler village $500 \mathrm{~m}$. 25.05.2006.

Pieris (Artogeia) ergane (Geyer, [1828])

Konya Akşehir sorkun 960m. 22.06.2004.

Pieris (Artogeia) rapae (Linnaeus, 1758)

Isparta Eğirdir Boyalıköy 900m. 19.05.2004; Ankara Batıkent Yuva village 900m. 02.07.2003; Ankara Ayaş Sinanl 1100m. 01.08.2003; Denizli Acıgöl vicinity 850m. 24.07.2003; Denizli Acıgöl vicinity 850m. 24.07.2003; Istanbul Şile Bozgoca village 30m. 21.07.2005; Istanbul Şile Bozgoca village 30m. 08.08.2005; Istanbul Şile Bozgoca village 50m. 25.08.2005; Konya Akşehir sorkun 960m. 22.06.2004; Konya Akşehir sorkun 960m. 02.07.2004; Nevşehir Hacıbektaş 1300m. 18.07.2004.

Pieris (Artogeia) pseudorapae Verity, 1908

Ankara Ayaş beli 1150m. 06.07.2004; Denizli Maymun Mountains Göz Location 900m. 20.07.2003; Istanbul Şile Bozgoca village 30m. 21.07.2005; Istanbul Şile Bozgoca village 30m. 08.08.2005; Konya Akşehir sorkun 960m. 22.06.2004; Konya Akşehir sorkun 960m. 02.07.2004; Malatya Akçadağ ikinciler village500m. 25.05.2006.

Pontia edusa (Fabricius, 1777)

Isparta Eğirdir Boyalıköy 900m. 19.05.2004; Ankara Batıkent Yuva village 900m. 02.07.2003; Ankara Ayaş Sinanl1 1100m. 01.08.2003; Denizli Maymun Mountains Göz Location 900m. 20.07.2003; Denizli Acıgöl vicinity 850m. 24.07.2003; Kırşehir Aşıkpaşa Atatürk forest 980m. 20.06.2004; Kırşehir Aşıkpaşa Atatürk forest $980 \mathrm{~m}$. 04.07.2004; Konya Akşehir sorkun 960m. 22.06.2004; Malatya Akçadağ ikinciler village500m. 25.05.2006.

Family: ARGYNNIDAE Duponchel, [1835]

Argynnis (Fabriciana) niobe (Linnaeus, 1758)

Kırşehir Aşıkpaşa Atatürk forest 980m. 27.06.2004; Malatya Akçadağ ikinciler village500m. 25.05.2006.

Argynnis (Pandoriana) pandora ([Denis \& Schiffermüller] , 1775)

Denizli Maymun Mountains Göz Location 900m. 20.07.2003; Denizli Acıgöl vicinity 850m. 24.07.2003.

Argynnis paphia (Linnaeus, 1758)

Antalya Thermossus National park 900m. 27.07.2003; Istanbul Şile Bozgoca village 30m. 21.07.2005; Istanbul Şile Bozgoca village 30m. 08.08.2005. 
Brenthis hecata ([Denis \& Schiffermüller], 1775)

Ankara Ayaş beli 1150m. 06.07.2004.

Issoria lathonia (Linnaeus, 1758)

Isparta Eğirdir Boyalıköy 900m. 19.05.2004; Kırşehir

Aşıkpaşa Atatürk forest 980m. 27.06.2004; Kırşehir Aşıkpaşa Atatürk forest 980m. 04.07.2004.

Limenitis reducta Staudinger, 1901

Denizli Maymun Mountains Göz Location 900m. 20.07.2003; Denizli Acıgöl vicinity 850m. 24.07.2003; Istanbul Şile Bozgoca village 30m. 08.08.2005.

Melitaea (phoebe) phoebe (Goeze, 1779)

Isparta Eğirdir Boyalıöy 900m. 19.05.2004; Ankara Ayaş Sinanl1 1100m. 01.08.2003.

Melitaea (Didymaeformis) didyma (Esper, [1779])

Ankara Ayaş beli 1150m. 06.07.2004; Istanbul Şile Bozgoca village 30m. 21.07.2005; Kırşehir Aşıkpaşa Atatürk forest 980m. 20.06.2004; Kırşehir Aşıkpaşa Atatürk forest 980m. 27.06.2004.

Melitaea (Didymaeformis) fascelis (Fabricius, 1787)

Isparta Eğirdir Boyalık̈y 900m. 19.05.2004.

Nymphalis antiopa (Linnaeus, 1758)

Kırşehir Aşıkpaşa Atatürk forest 980m. 27.06.2004;

Nevşehir Hacıbektaş 1300m. 18.07.2004.

Polygonia (Comma) c-album (Linnaeus, 1758)

Ordu Fatsa Baba village 500m. 28.08.2006.

Vanessa cardui (Linnaeus, 1758)

Isparta Eğirdir Boyalıöy 900m. 19.05.2004; Ankara

Batıkent Yuva village 900m. 02.07.2003; Ankara Ayaş Sinanl1 1100m. 01.08.2003; Denizli Maymun Mountains Göz Location 900m. 20.07.2003; Denizli Acıgöl vicinity 850m. 24.07.2003; Istanbul Şile Bozgoca village $30 \mathrm{~m}$. 21.07.2005; Konya Akşehir sorkun 960m. 22.06.2004; Konya Akşehir sorkun 960m. 02.07.2004; Ordu Fatsa Baba village 500m. 28.08.2006.

Family: SATYRIDAE Boisduval, 1833

Brintesia circe (Fabricius, 1755)

Ankara Ayaş beli 1150m. 06.07.2004.

Chazara (Neochazara) anthe (Hoffmannsegg, 1804)

Kırşehir Aşıkpaşa Atatürk forest 980m. 20.06.2004;

Kırşehir Aşıkpaşa Atatürk forest 980m. 27.06.2004;

Kırşehir Aşıkpaşa Atatürk forest 980m. 04.07.2004.

Chazara briseis (Linnaeus, 1764)

Ankara Batıkent Yuva village 900m. 02.07.2003; Ankara

Ayaş Sinanl 1100m. 01.08.2003; Ankara Ayaş beli 1150m. 06.07.2004; Denizli Acıgöl vicinity 850m. 24.07.2003;

Kırşehir Aşıkpaşa Atatürk forest 980m. 27.06.2004;

Kırşehir Aşıkpaşa Atatürk forest 980m. 04.07.2004;

Nevşehir Hacıbektaş 1300m. 18.07.2004.

Chazara bischoffii (Herrich-Schaffer, [1846])

Kırşehir Aşıkpaşa Atatürk forest 980m. 27.06.2004;

Kırşehir Aşıkpaşa Atatürk forest 980m. 04.07.2004.

Coenonympha pamphilus (Linnaeus, 1758)

Isparta Eğirdir Boyalıköy 900m. 19.05.2004; Ankara Ayaş Sinanl 1100m. 01.08.2003; Malatya Akçadağ ikinciler village $500 \mathrm{~m}$. 25.05.2006; Ordu Fatsa Baba village 500m. 28.08.2006.

Hipparchia (Neohipparchia) fatua (Freyer, 1844)
Denizli Acıgöl vicinity 850m. 24.07.2003.

Hipparchia (Parahipparchia) aristaeus (Bonelli, 1826)

Ankara Ayaş Sinanlı 1100m. 01.08.2003; Denizli Maymun Mountains Göz Location 900m. 20.07.2003.

Hipparchia (Parahipparchia) mersina (Staudinger, 1871) Antalya Thermossus National park 900m. 27.07.2003.

Hipparchia (Parahipparchia) pellucida (Stauder, 1924)

Denizli Acıgöl vicinity 850m. 24.07.2003.

Hipparchia syriaca (Staudinger, 1871)

Istanbul Şile Bozgoca village 30m. 08.08.2005.

Hyponephele lupina (Costa, [1836])

Antalya Thermossus National park 900m. 27.07.2003; Ankara Ayaş beli 1150m. 06.07.2004; Kırşehir Aşıkpaşa Atatürk forest 980m. 20.06.2004; Kırşehir Aşıkpaşa Atatürk forest $980 \mathrm{~m}$. 27.06.2004.

Krinia (Melike) roxelana (Cramer, [1777])

Ankara Ayaş beli 1150m. 06.07.2004.

Lasiommata megera (Linnaeus, 1767)

Ankara Ayaş Sinanl1 1100m. 01.08.2003; Istanbul Şile Bozgoca village $30 \mathrm{~m}$. 21.07.2005; Istanbul Şile Bozgoca village 50m. 25.08.2005.

Maniola (Telmessiola) telmessia (Zeller, 1847)

Ankara Batıkent Yuva village 900m. 02.07.2003; Istan-

bul Şile Bozgoca village 50m. 25.08.2005.

Maniola jurtina (Linnaeus, 1758)

Antalya Thermossus National park 900m. 27.07.2003; Ankara Ayaş Sinanlı 1100m. 01.08.2003; Ankara Ayaş beli 1150m. 06.07.2004; Istanbul Şile Bozgoca village $30 \mathrm{~m}$. 08.08.2005; Ordu Fatsa Baba village 500m. 28.08.2006; Kırşehir Aş1kpaşa Atatürk forest 980m. 27.06.2004.

Melanargia (Turcargia) larissa (Geyer, [1828])

Ankara Ayaş beli 1150m. 06.07.2004; Kırşehir Aşıkpaşa Atatürk forest 980m. 27.06.2004; Kırşehir Aşıkpaşa Atatürk forest $980 \mathrm{~m} .04 .07 .2004$.

Melanargia galathea (Linnaeus, 1758)

Istanbul Şile Bozgoca village 30m. 21.07.2005.

Pararge aegeria (Linnaeus, 1758)

Ordu Fatsa Baba village 500m. 28.08.2006.

Pseudochazara (Achazara) anthelea (Hübner, [1824])

Antalya Thermossus National park 900m. 27.07.2003;

Kırşehir Aşıkpaşa Atatürk forest 980m. 20.06.2004;

Kırşehir Aşıkpaşa Atatürk forest 980m. 27.06.2004;

Nevşehir Hacıbektaş 1300m. 18.07.2004.

Pseudochazara lydia (Staudinger, 1878)

Kırşehir Aşıkpaşa Atatürk forest 980m. 20.06.2004;

Kırşehir Aşıkpaşa Atatürk forest 980m. 04.07.2004.

Pseudochazara mniszechii (Herrich- Schäffer , [1851])

Ankara Batıkent Yuva village 900m. 02.07.2003;

Kırşehir Aşıkpaşa Atatürk forest 980m. 27.06.2004;

Kırşehir Aşıkpaşa Atatürk forest 980m. 04.07.2004.

Pyronia tithonus (Linnaeus, 1771)

Istanbul Şile Bozgoca village 30m. 21.07.2005.

Family: LYCAENIDAE Stephenes, 1829

Celastrina argiolus (Linnaeus, 1758)

Antalya Thermossus National park 900m. 27.07.2003.

Cupido osiris (Meigen, [1829])

Isparta Eğirdir Boyalıköy 900m. 19.05.2004. 
Cupido minimus (Fuessly, 1775)

Isparta Eğirdir Boyalıköy 900m. 19.05.2004.

Glaucopsyche (Apelles) astraea (Freyer, [1851])

Isparta Eğirdir Boyalıköy 900m. 19.05.2004.

Glaucopsyche alexis (Poda, 1761)

Isparta Eğirdir Boyalıöy 900m. 19.05.2004.

Lampides boeticu (Linnaeus, 1767)

Ankara Ayaş Sinanl1 1100m. 01.08.2003; Ankara Ayaş beli 1150m. 06.07.2004; Kırşehir Aşıkpaşa Atatürk forest 980m. 27.06.2004; Nevşehir Hacıbektaş 1300m. 18.07.2004.

Lycaena (Thersamonia) thersamon (Esper, [1784])

Ankara Ayaş Sinanlı 1100m. 01.08.2003; Kırşehir Aşıkpaşa Atatürk forest 980m. 20.06.2004.

Lycaena phlaeas (Linnaeus, 1761)

Ankara Batıkent Yuva village 900m. 02.07.2003; Ankara Ayaş Sinanlı 1100m. 01.08.2003; Ordu Fatsa Baba village 500m. 28.08.2006.

Plebejus (Kretania) carmon (Gerhard, [1851])

Denizli Acıgöl vicinity 850m. 24.07.2003; Kırşehir Aşıkpaşa Atatürk forest 980m. 27.06.2004.

Plebejus (Lycaeides)idas (Linnaeus 1761)

Denizli Acıgöl vicinity 850m. 24.07.2003; Kırşehir Aşıkpaşa Atatürk forest 980m. 20.06.2004; Kırşehir Aşıkpaşa Atatürk forest 980m. 27.06.2004.

Plebejus (Plebejides) sephirus (Frivaldzky, 1835)

Kırşehir Aşıkpaşa Atatürk forest 980m. 20.06.2004;

Kırşehir Aş1kpaşa Atatürk forest 980m. 27.06.2004.

Polyommatus (Albulina) loewii (Zeller, 1847)

Kırşehir Aşıkpaşa Atatürk forest 980m. 20.06.2004;

Kırşehir Aş1kpaşa Atatürk forest 980m. 27.06.2004.

Polyommatus (Aricia) anteros (Freyer, [1838])

Istanbul Şile Bozgoca village 50m. 25.08.2005.

Polyommatus (Aricia) agestis ([Denis \& Schiffermüller], 1775)

Antalya Thermossus National park 900m. 27.07.2003;

Kırşehir Aşıkpaşa Atatürk forest 980m. 27.06.2004.

Polyommatus (Agrodiaetus) menalcas (Freyer, [1837])

Ankara Batıkent Yuva village 900m. 02.07.2003; Denizli

Maymun Mountains Göz Location 900m. 20.07.2003.

Polyommatus (Lysandra) bellargus (Rottemburg, 1775)

Malatya Akçadağ ikinciler village500m. 25.05.2006.

Polyommatus (Plebicula) amandus (Schneider, 1792)

Antalya Thermossus National park 900m. 27.07.2003;

Isparta Eğirdir Boyalıköy 900m. 19.05.2004.

Polyommatus (Thersitesia) thersites (Canterer, [1835])

Isparta Eğirdir Boyalıköy 900m. 19.05.2004; Ankara

Ayaş Sinanl 1100m. 01.08.2003; Kırşehir Aşıkpaşa Atatürk forest $980 \mathrm{~m}$. 27.06.2004.

Polyommatus icarus Rottemburg, 1775

Isparta Eğirdir Boyalıköy 900m. 19.05.2004; Ankara Ayaş Sinanlı 1100m. 01.08.2003; Ankara Ayaş beli 1150m. 06.07.2004; Denizli Maymun Mountains Göz Location 900m. 20.07.2003; Denizli Acıgöl vicinity $850 \mathrm{~m}$. 24.07.2003; Istanbul Şile Bozgoca village 30m. 21.07.2005; Kırşehir Aşıkpaşa Atatürk forest 980m. 27.06.2004; Konya Akşehir sorkun 960m. 22.06.2004; Konya Akşehir sorkun 960m. 02.07.2004; Nevşehir Hacıbektaş 1300m. 18.07.2004; Malatya Akçadağ ikinciler village $500 \mathrm{~m}$. 25.05.2006; Ordu Fatsa Baba village 500m. 28.08.2006.

Pseudophilotes vicrama (Moore, 1865)

Kırşehir Aşıkpaşa Atatürk forest 980m. 20.06.2004.

Polyommatus (Agrodiaetus) wagneri (Forster, 1956)

Nevşehir Hacıbektaş 1300m. 18.07.2004.

Satyrium (Nordmannia) acaciae (Fabricius 1787)

Isparta Eğirdir Boyalıköy 900m. 19.05.2004.

Superfamily: HESPERIOIDEA Latreille, 1809

Family: HESPERIIDAE Latreille, 1809

Erynnis (Hesperopegasus) marloyi (Boisduval, [1834])

Denizli Maymun Mountains Göz Location $900 \mathrm{~m}$. 20.07.2003.

Hesperia comma (Linnaeus, 1758)

Istanbul Şile Bozgoca village 30m. 21.07.2005.

Pyrgus melotis (Duponchel, [1834])

Isparta Eğirdir Boyalıköy 900m. 19.05.2004.

Spialia (Neaspialia) orbifer (Hübner, [1823])

Kırşehir Aşıkpaşa Atatürk forest 980m. 20.06.2004.

Thymelicus lineolus (Ochsenheimer, 1808)

Ankara Batıkent Yuva village 900m. 02.07.2003;

\section{Discussion}

The butterfly population has decreased or vanished due to the destruction of agricultural areas and urbanization, and accordingly, the disintegration and loss of habitats (Avc1, 1994). For proper and efficient sampling, Lepidoptera species were collected in different localities possessing, different vegetation types (agricultural crops to Pinus and Quercus forests) and altitudes from 30 to $1300 \mathrm{~m}$. In this study it is clearly all these characteristics (locality, vegetation type, altitude and date) have an effect on distribution of living organisms, including butterflies.

In this study 77 species belonging to 6 families from order Lepidoptera identified. These families and number of species are: Papilionidae (3); Pieridae (13); Argynnidae (12); Satyridae (22); Lycaenidae (22) and Hesperiidae (5).

I.podalirius; P. machaon; C. crocea; P. brassicae; P. rapae; P. pseudorapae; P. edusa; V. cardui; C. briseis; M. jurtina and $P$. icarus are the species were determined as widespread.

This study makes contribution to the Lepidoptera fauna of Ankara, Antalya, Denizli, Isparta, Istanbul, Kırşehir, Konya, Malatya, Nevşehir and Ordu in Turkey. Scientific and taxonomic studies on Lepidoptera in Anatolia are very limited in number. New studies considering insect orders should be carried out before agricultural and forest ecosystems are destroyed. On the other hand, the continuing illegal use of forests and incorrect land use practices threaten to destroy the diversity in Anatolia. Therefore, detailed studies should be carried out as quickly as possible to determine the biological diversity in Turkey.

\section{References}


[1] Atay E, Yolcu S, "Butterfly fauna of the province of Hatay, Turkey and major taxonomic characters of Polyommatus bollandi Dumont, 1998 (Lycaenidae)". Pakistan J. Zool. 2012, 44: 893-896.

[2] Ayberk H, Hakyemez A, Cebeci H, "Light trap surveys for moths in Sile region of Istanbul, Turkey". Afri. J. of Biotech. 2010. 9()29, pp. 4624-4630.

[3] Can F, "The Geometrid Moths (Lepidoptera) from the Middle and Eastern Black Sea Regions of Turkey". Turk J. Zool. 2008, 32: 351-358

[4] Cassie B, "A world of Butterflies". First Edition Third Printing, Chanticleer Press, Inc., and Charles Nix \& Assoc. Hachette Book Group USA, 237 Park Avenue, New York, 2007, 420pp.

[5] Hesselbarth G, Van Oorschot H, Wagener S, "Die Tagfalter der Turkei, 3 band." Selbstverlag Sigbert Wagener Hemdener Weg 19, D 46399 Bocholt, Germany, 1995.

[6] Hofmann H, Marktanner T,'Butterflies and Moths of Britain \& Europe". Collins Nature Guides. Harpur Collins Publishers, London. 1995, 158pp.

[7] Hüseyinoğlu Y, "Sincanlı (Afyon) Papilionoidea ve Hesperioidea Faunası Üzerine Araştırmalar". Cent. ent. Stud., Miscellaneous Papers. 2000, 60: 1-8.

[8] Karaçetin E, Welch HJ, "Türkiye'deki Kelebeklerin Kırmızı Kitabı”. Ankara: Doğa Koruma Merkezi. 2011, www.dkm.org.tr.

[9] Kaygin AT, Yildiz Y, Avci M, "Lepidoptera fauna in Bartin Province, in western black sea region of Turkey". Afr. J. Agric. Res. 2009, 4(9): 815-822.
[10] Koçak AÖ, Seven S, “Türkiye Lepidoptera Bibliyografyası". Cent. ent. Stud., Memoirs, 1994, 2: 1-141.

[11] Koçak AÖ, Seven S, “Tokat Papilionoidea ve Hesperioidea Faunası ve Ekolojisi Hakkında (Lepidoptera)". Cent. ent. Stud., Priamus 1995, 7 (3): 93-116.

[12] Koçak AÖ, Kemal M, "Checklist of the Diurnal Lepidoptera of Turkey". Cent. ent. Stud., Priamus Supplement, 2008, $15: 1-42$.

[13] Koçak AÖ, Kemal M, "Revised Checklist of the Lepidoptera of Turkey". Cent. ent. Stud., Priamus Supplement, 2009, $17: 1-253$

[14] Koçak AÖ, Kemal M, "List of the Hitherto recorded Pterygot taxa of Turkey (Insecta)". Cent. Ent. Std., Memoris 6. 2012.

[15] Kudrna O, "An annotated catalogue of the butterflies named by Roger” Verity. J. Res. Lepid. 1983, 21: 1-106.

[16] Lederer J, "Beitrag zur Schmetterlings-Fauna von Cypern, Beirut und einem Theile Klein-Asiens". Verh. zool.- bot. Ver. Wien, 1855, 5: 177-254, 5 Taf.

[17] Schappert P, "A World for Butterflies, Their Lives, Behavior and Future". Published by Firefly Books (U. S.) Inc. First Printing, 2005, 320pp.

[18] Okyar Z, Aktac N, "Additives to Heterocera (Lepidoptera) fauna of Thracian Region". Turkish J. Entomol. 1998, 22(1): $47-56$

[19] Tolman T, Lewington R, "Butterflies of Britain \& Europe. Collins field Guide". Harper Collins Publishers. London, 1997, 320pp. 